\title{
Progressive AODV: A Routing Algorithm Intended for Mobile Ad-Hoc Networks
}

\author{
M. Sakthivel, J. Udaykumar, V. Saravana Kumar
}

\begin{abstract}
Mobile ad hoc networks are an independent wireless network that is built without permanent infrastructure and base station support. Each node in the network uses a wireless connection for connections and acts not only as an end system but also as a path to accelerating packets. Meanwhile, the network nodes are mobile and can move in each path with changing steps, creating a great dynamic of the network. Therefore, the protocols set for general ad hoc networks are inappropriate for such a situation. In addition, the performance of ad hoc routing protocols decreases with increasing network size. In this context, suggest a new way to extend the recital of routing in MANETs: the on-demand Progressive Distance Vector (PAODV). It is a modified variant of the standard AODV routing protocol, which shrinks the dynamic path when an optimal path exists and switches the traffic to it. Simulation studies with NS2 show that the proposed method improves network performance as network size, weight, or flexibility increase.
\end{abstract}

Keywords: MANETs, AODV, Routing protocols.

\section{INTRODUCTION}

These days, due to the quick growth of wireless communication ground, a generation of Ad-hoc networks, Mobile Ad-hoc network has expanded cumulative consideration of the researchers. MANETs is a wireless network, vigorously establishing a communication network without any consolidated control and already existing network structure.

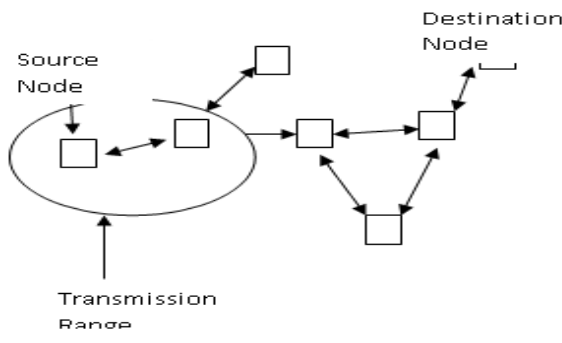

Figure: 1 Mobile ad-hoc network with seven nodes

Revised Manuscript Received on December 30, 2019.

* Correspondence Author

M. Sakthivel*, (Correspondence Author) Department of CSE, Sree Vidyanikethan Engineering College, Tirupati (A.P), India.

J. Udaykumar, Department of CSE, Sree Vidyanikethan Engineering College, Tirupati (A.P), India.

V. Saravana Kumar, Department of IT, Sreenidhi Institute of Science and Technology, Hyderabad, Telangana, India,

(C) The Authors. Published by Blue Eyes Intelligence Engineering and Sciences Publication (BEIESP). This is an open access article under the CC BY-NC-ND license (http://creativecommons.org/licenses/by-nc-nd/4.0/)
As the nodes are Mobiles in MANETs, alteration their positions quickly, so discovery a transport path to an endpoint is a stimulating task. On the other hand, some exclusive character of such network like regular changes in topology, flexibility patterns, changing density over time, channel fading, and unbalanced communication situations pose several exclusive investigation challenges for routing protocols actuality used in MANETs. Apart from these, the speed and dimensions of the network lower the presentation of routing protocols and pose novel tasks in front of researchers to design a well-organized routing algorithm for MANETs situation [1-5]. Though a figure of exclusive methods has been projected by numerous of researchers in last few decades to overwhelmed the routing matters of MANETs, still, no one routing method is effective to outperform in all situations of ad-hoc networks.

Every projected answer has its exclusive advantages in some definite networking backgrounds, but Mobile nodes should be able to function in every neighbour that pose experiment to researchers regarding strategy an effective routing procedure [6-8]. The effort described in this paper discourse the routing subjects of an available routing protocol in surroundings of MANETs and announces enhanced routing answer which increases the routing concert in stimulating an environment of MANETs. The rest of the paper is structured as follows: Section II presents the classification of various routing protocols. Sections III present the related work. The issues of ad-hoc routing protocols present in section IV. Proposed method present in section V. Section VI present simulation results and analysis work and finally conclusion and future work present in section VII.

\section{ORGANIZATION OF ROUTING PROTOCOLS}

The communication presentation of a network depends on how the location of the message in the path improves. Classically routing is the act of transferring data from a source to a destination in an Internetwork. Routing protocols allow network nodes to choose paths between different pairs of nodes for the data exchange process, using network participants in between to promote packets on their way to the destination. Since that time, the age of MANETs, various ad hoc routing protocols have been estimated, has been discussed. [9-11] Although these protocols can be classified in many ways, routing protocols for MANETs can be classified into three main types, such as routing plans, network construction and rendering for the area / application where they are most suitable. That is shown in Figure 2. 


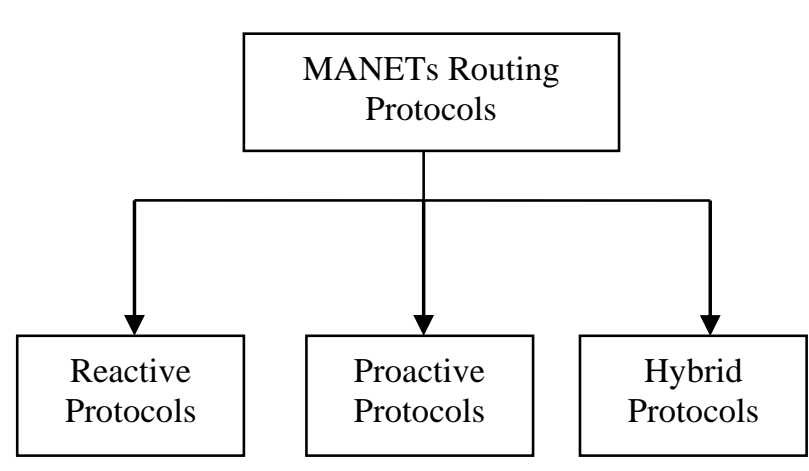

Figure: 2 Categories of MANETs Routing Protocols

\section{A. Proactive Routing Protocols}

Proactive routing protocols are typically built on a shortest path algorithm and are identified as table-driven routing protocols because they stock the data of all related nodes in the process of tables. This type of routing protocol maintains paths for all endpoints, whether or not these routes are necessary. Each time multiple change nodes present in the network shared information with their partner nodes. To maintain the correct path data, a node needs to induce control messages every time. As a result, proactive routing protocols can waste bandwidth because control messages are sent improperly when there is no data traffic flow.

\section{B. Reactive Routing Protocols}

Routing protocols that drop under reactive routing protocols are also well-known as on-demand routing protocols. Reactive / on-demand routing protocols establish a relationship for a pair of nodes only when it is required and only for those nodes that are currently being used to send data packets from point to point. , Thus reducing the overhead problem because proactive routing protocols. Although reactive-type protocols reduce the cases of active routing protocols, they use a flooding process to detect the path, which causes more route overheads, bandwidth ingestion, and battery powers. Apart from these, protocols are also stimulated by the original path detection process, which makes them unsuitable for care presentations in MANETs.

\section{Hybrid Routing Protocols}

Cross routing protocol associations are characteristics of both reactive and active passive protocols. These groups of protocols are declared to trim the initial path tracing the overhead ratio and delay of the remaining routing protocols. Naturally, cross-routing protocols are region-based protocols where the number of nodes is divided into different regions. But, while cross routing protocols simultaneously cover the functionality of reactive and proactive routing protocols and reduce the cases of current processes, protocols based on cross technology face the difficulty of network connectivity as an active in a large network situation. Its mechanism like passive protocol, and in a small system it behaves like a reactive routing protocol so that small nodes are not fixed under conditions through a density network.

\section{CORRELATED WORK}

Then the arrival of MANET, planning, and application of an efficient routing protocol with good performance and low overhead is one of the fundamental challenges of this network. While there has been a lot of research en route for
MANET to trim such routing issues for real-time network conditions, specific appearances of such networks such as high mobility, rapid changes in network topology, scalability, any Routing is not Protocol is a way to outstrip every state of MANETs. Many academics [12-20] have observed the applicability of customary defeating protocols in environments of MANETs by large-scale tests. They have associated the AODV routing protocol with a number of methods at the beginning of propagation numbers and system conditions to illustrate the effectiveness of such protocols. However, he was engrossed in reassuring aspects of the simulation. Their test results require that the AODV routing protocol is more suitable for the energetic location of MANETs, but there are some drawbacks such as large overheads in large networks, using high-energy ingestion and flooding processes that generate the need for adjustment.

In the direction of reducing the network's energy ingestion relationship and enhancing link stability under the high active state of MANETs, the authors [21] have estimated EEAODR, a novel energy-efficient on-demand routing algorithm. The proposed method extends the working techniques of the traditional AODV routing scheme and focuses on maintaining the energy balance between network nodes to enhance network stability. By modifying the path establishment technique of existing AODV routing protocols in the same direction, a novel energy-effective routing strategy is accessible in [22].

The approximate method determines the optimal path based on the maximum energy of each path. The proposed method has its utility on the out-of-date routing system of test results. In [23], the authors have announced a new, improved type of conservative AODV routing protocol, called EODV. The approximate method rewards the actual stretch VoIP's QoS in wireless ad hoc systems by overriding the untouched part of the web. Virtual results have presented the effectiveness of the EAODV protocol over the old-fashioned routing method. In a similar way, a new technique is planned [24], named A-SAODV (Adaptive SAODV), to recover the presentation of conservative AODV routing protocols. The method tunes the behaviour of SAODV by incorporating filtering strategies.

In [25], the authors propose a neighbor trust that is based on a customized routing algorithm to extend the text of routing in the case of MANETs. The method modified the path request packet of the traditional AODV routing protocol by computing an additional field that stores the neighbor trust value. For the pack distribution, the approximate method has used the extreme confidence value of the neighbor. Thus it not only conserves the power of the node by passing redundant program controller evidence, but also improves the course-plotting statement in relation to bandwidth (channel usage), which is important in the case of MANET. Several other procedures [26-28] have also suggested towards reducing routing problems of MANET.

\section{DISQUIETS OF AD-HOC ROUTING PROTOCOLS}

However, there are many modifications since the phases of the network and have been conducted to propose more streamlined routing protocols and they improve routing statements due to disagreement 
constraints but difficulty in doing the right thing and maintaining the correct paths Many of the earlier treaties improve with. For endpoints through dynamics and moving topology. In cache, the proposed methods have a routing route at the forefront that uses upstream and additional bandwidth and nodes control in the declaration, performing different tasks for routing different ground MAN MANs in a higher dynamic setting. In MANETs, the topics of the available routing protocols can be indicated as points.

- When node residents are small, manageable routing protocols simply operate.

- Proactive routing protocols will be overwhelmed by rapid topology fluctuations and even fail to break through the routing information exchange phase.

- The reactive routing structure will fail to realize the entire path due to multiple network partitions.

- Current routing protocols use the inducting process to arrange a connection between a pair of nodes, so great bandwidth is swallowed and produces high-end to end-times.

- Squat scalability, where the network can sometimes go from rough to very short.

\section{PROJECTED METHOD}

Subsequently, the nodes are movable in the MANET, changing with rapidly changing velocity and density. Nevertheless, the high resilience of the network is the central core of the quick association disaster, but because of this resilience, there is an indication that two or additional nodes, which are a part of the energetic pathway, originate in each other without violating the current message. Straight variety. The deprecated AODV routing protocol does not reflect this condition. This packet demonstrates the active route for the advance, unless there is a connection. In this event the packet mobile increases the distance to heavy distances and the possibility of dropping and network crashes, although the ideal direct route is available. This functionality includes the presentation of routing protocols that use reality in the network to route packets.

In this case, a new routing method called progressive AODV, to increase the routing representation and scalability of MANET networks. The proposed method is an illuminated version of the ad-hoc on-demand distance vector (AODV) routing protocol. The technique employed includes the self-healing routing process with an out-of-date AODV routing protocol to reduce swag, improve the presentation of the departure method in accrual over assets, and reduce the collaboration of the planned technique. The technique is to check the active route seamlessly and every time a shortcut path exists, which does not include nodes determined by the live path, repeated reading of the route reduces mobility with multiple hops.

\section{SIMULATION RESULTS AND ANALYSIS}

\section{A. Simulation Tool \& Parameters}

To appraise the presentation of projected technique, quite a lot of-of simulations are directed by consuming the Network Simulator NS-2.32 [16] with changing network weight from small to great, variable mobility and variation the speediness of network nodes.

Table: 1 summarized simulation parameters.

\begin{tabular}{|l|l|}
\hline \multicolumn{1}{|c|}{ Parameters } & \multicolumn{1}{c|}{ Values } \\
\hline Simulation Area & $1000 \mathrm{~m} \mathrm{X} \mathrm{1000} \mathrm{m}$ \\
\hline Number of Nodes & $20,40,60,80,110$ \\
\hline Pause Time & $10 \mathrm{~ms}$ \\
\hline Transmission Range & $200 \mathrm{~m}$ \\
\hline MAC Protocol & IEEE 802.11 \\
\hline $\begin{array}{l}\text { Vehicle } \\
\text { (Maximum) }\end{array}$ & $20 \mathrm{~m} / \mathrm{s}$ \\
\hline Simulation Time & 200 sec. \\
\hline Traffic & Constant Bit Rate(CBR) \\
\hline Packet Size & 512 bytes \\
\hline Queue type & priQueue \\
\hline Routing Protocol & AODV, P_AODV \\
\hline
\end{tabular}

\subsection{Results Analysis}

Virtual reality effects have been examined by likening the presentation metrics of Throughput, Packet Distribution Ratio (PDR), Stabilized Routing Capacity (SRC) and the End to End Deferrals (E2ED).

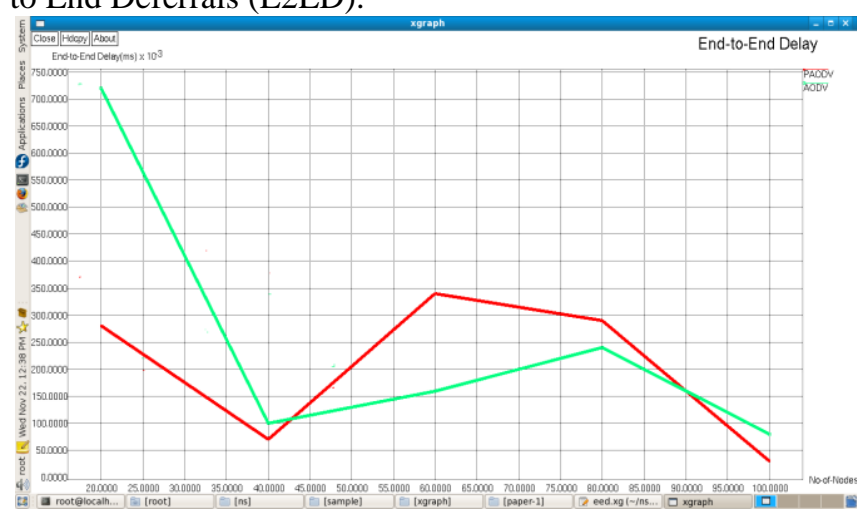

- Average End to End Delay (E2E): The end to end deferral (E2E) metric offerings the normal time engaged by the packets to permit through the network, effectively transported to their endpoints.

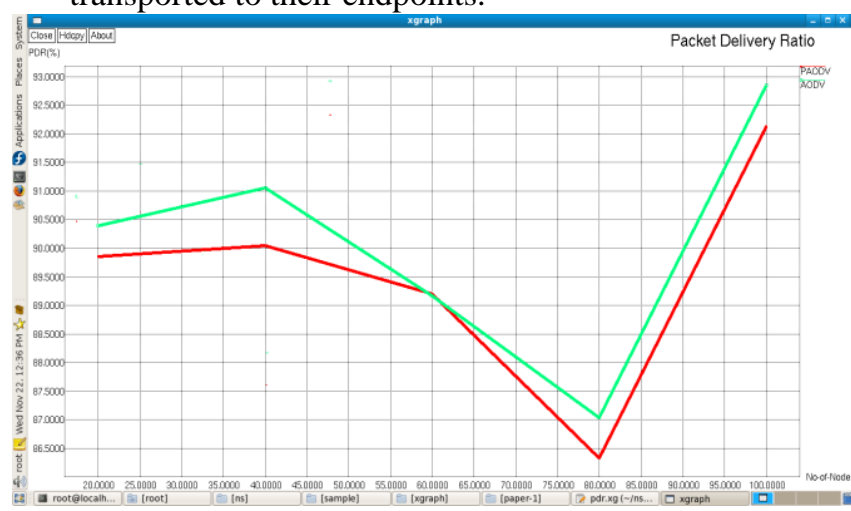

- $\quad$ Packet Distribution Ratio (PDR): This metric provides the proportion of the entire data packets positively established at the endpoint and an entire number of data packets produced at starting point. 


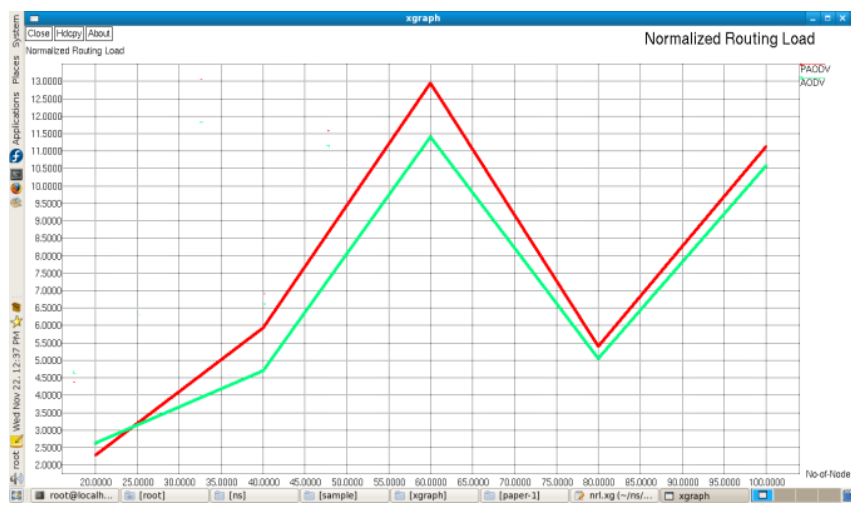

- Stabilized Routing Capacity (SRC): Stabilized Routing capacity is the records of routing packets conveyed per data packet directly to the endpoint.

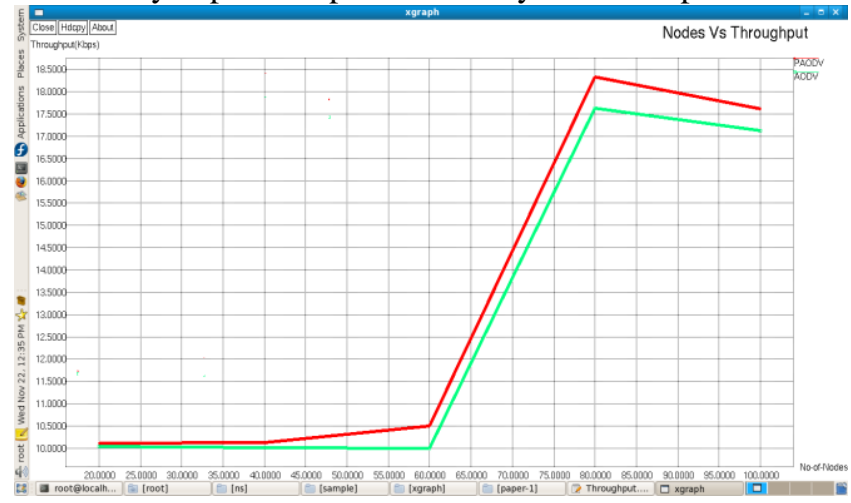

- Throughput: The throughput of the protocols can be demarcated as the ratio of the packets acknowledged by the endpoint between the packets directed to the starting point. It is the quantity of data per time unit that is transported from one node to alternative via an announcement association. The throughput is dignified in bits per second.

\section{CONCLUSION \& FUTURE WORK}

This paper discourse the routing matters of MANETs and projected an innovative routing technique by improving the route repairs technique of out-of-date AODV routing protocol, aim to deteriorate the routing presentation in the stimulating situation of MANETs. The projected method has display dynamic routing routes uninterruptedly and transmitting the Route every time a shortcut route is available. It eliminates the redundant nodes from the path. Quite a few of simulation approved out to existing the effectiveness of a planned technique by variable the circulation burden and flexibility in the network. The simulation outcomes obviously specify the productivity and usefulness of projected technique done the out-of-date AODV routing protocol. Consequently, coming trepidations added examination is required regarding offer power regulator in the infrastructures. For wireless networks, energy is a continuously vigorous resource. In Accumulation forthcoming work can be completed in a way to sleek down the percentage of End to End deferrals. It can enrich the recitation of the network in a novel approach.

\section{REFERENCES}

1. Mehmood, Zafar, Muddesar Iqbal, and Xinheng Wang. "Comprehensive experimental performance analysis of DSR, AODV and DSDV routing protocol for different metrics values with predefined constraints." International Journal of Information technology and computer science, vol. 6, no.7, pp: 24-31, 2014.

2. Sakthivel, Muthusamy, and Veerappa Gounder Palanisamy. "Enhancement of accuracy metrics for energy levels in MANETs." Computers \& Electrical Engineering,vol. 48, pp: 100-108, 2015.

3. Minh, Thu Pham Thi, Trong Tien Nguyen, and Dong-Seong Kim. "Location aided zone routing protocol in mobile Ad Hoc Networks." 2015 IEEE 20th Conference on Emerging Technologies \& Factory Automation (ETFA). IEEE, 2015.

4. Sharma, Ramnik, and Anita Kumari. "A Review on Traffic Route Optimizing by Using Different Swarm Intelligence Algorithm." International Journal of Computer Science and Mobile Computing, vol. 4, no.5, pp: 271-277, 2015.

5. Chen, YouRong, et al. "Power control routing algorithm for maximizing lifetime in wireless sensor networks." Advances in Mechanical and Electronic Engineering. Springer, Berlin, Heidelberg, pp:129-136, 2013.

6. Ramanathan, Subramanian. "A unified framework and algorithm for channel assignment in wireless networks." Wireless Networks, vol.5, no.2, pp: 81-94, 1999

7. Syed, S. Syes Abdul, and T. Senthil Kumaran. "Extremely Vibrant Routing Scheme for Mobile Adhoc Network." Indonesian Journal of Electrical Engineering and Computer Science, vol. 9, no.2, pp: 306-310, 2018.

8. Manikandan, E., S. Muthukumarasamy, and K. Thanigaivelu. "Stratified Report Assisted Reputation Administration (SRA) system for MANETs." 2016 International Conference on Information Communication and Embedded Systems (ICICES). IEEE, 2016.

9. Kumar, Sushil, and Anil Kumar Verma. "Position based routing protocols in VANET: a survey." Wireless Personal Communications, vol. 83, no.4, pp: 2747-2772, 2014.

10. Singh, Vijander, and Reena Dadhich. "Routing optimization by minimizing one-way delay in delay tolerant enabled vehicular ad-hoc networks using ferry selection approach." 2017 International Conference on Infocom Technologies and Unmanned Systems (Trends and Future Directions)(ICTUS), IEEE, 2017.

11. Tyagi, Radheshyam, Sanjay Singh Kushwah, and Ashraf Samarah. "Competent Routing Protocol in Directional MAC for Handling Deafness in Ad Hoc Networks." 2015 Fifth International Conference on Communication Systems and Network Technologies, IEEE, 2015.

12. Paliwal, K. K., Surjeet Singh, and Suman Bajaj. "Rural communication enhancement using mobile ad-hoc network." 2017 7th International Conference on Cloud Computing, Data Science \& Engineering-Confluence. IEEE, 2017.

13. Walia, Himanshu, ErMandeep Singh, and Rahul Malhotra. "A Review: Mobile AdQ Hoc Routing Protocols." International Journal of Future Generation Communication and Networking, vol. 9, no.2, pp: 193-198, 2016.

14. Kumar, K. Pradeep, and BR Prasad Babu. "CIANI: Cognitive Intelligence for Assessing Network Intrusion in Mobile Adhoc Network." Wireless Personal Communications, pp : 1-19, 2019.

15. Priya, S. Banu, and C. Theebendra. "A Study On Security Challenges In Mobile Adhoc Networks." International journal of research in computer applications and robotics, pp: 2320-7345, 2016.

16. Jain, Rachna, and Indu Kashyap. "An QoS Aware Link Defined OLSR (LD-OLSR) Routing Protocol for MANETS." Wireless Personal Communications,pp: 1-14, 2019.

17. Devi, Munisha, and Nasib Singh Gill. "Study of Mobile Ad hoc Network Routing Protocols in Smart Environment." International Journal of Applied Engineering Research, vol. 13, no.16, pp: 12968-12975, 2018.

18. Maragathasundari, S., and K. S. Dhanalakshmi. "Mobile ad hoc networks problem-a queueing approach." International Journal of Communication Networks and Distributed Systems, vol. 21, no.4, pp: 475-495, 2018.

19. Kumar, Sanjay, and Dr Sudhir Kumar Rathi. "A Review: Novel Protocol for Clumping in Mobile Ad hoc Networks." International Journal of Innovative Research in Computer and Communication Engineering, vol. 3, no.4, 2015.

20. Shenbagapriya, S., and Ms N. Radhika. "Energy Power Aware Routing Protocol To Maximize Network Life Time In Manet." ENERGY, vol.4, no.5, 2015. 
21. Reji, M., et al. "Performance Metrics of Wormhole Detection using Path Tracing Algorithm." Indian Journal of Science and Technology, vol. 8, no.17, pp: 63541, 2015.

22. Choudhary, Sapna, and Sonal Jain. "A survey of energy-efficient fair routing in MANET." International Journal of Scientific Research in Science, Engineering and Technology,pp: 416-421, 2015.

23. Zhao, Nan, Feng Zeng, and Wenjia Li. "An Effective Routing Algorithm Based on Social Community for Mobile Opportunistic Networks." Proceedings of the 10th EAI International Conference on Mobile Multimedia Communications. ICST (Institute for Computer Sciences, Social-Informatics and Telecommunications Engineering), 2017.

24. Singh, Sushama, Atish Mishra, and Upendra Singh. "Detecting and avoiding of collaborative black hole attack on MANET using trusted AODV routing algorithm." 2016 Symposium on Colossal Data Analysis and Networking (CDAN). IEEE, 2016

25. Sangeetha, J., et al. "An analysis and comparison of different routing algorithms in WiMAX networks." 2015 IEEE International Advance Computing Conference (IACC). IEEE, 2015.

26. Junfeng, Tian. "A Study of Wireless Ad-hoc Network Protocol using Composite Routing Distance Algorithm." International Journal of Simulation--Systems, Science \& Technology, vol.17, no.36, 2016.

27. Emami, A. Babak, et al. "SNACK: An efficient intrusion detection system in Mobile Ad-Hoc Network based on the Selective-Negative Acknowledgement algorithm." 2015 IEEE 28th Canadian Conference on Electrical and Computer Engineering (CCECE). IEEE, 2015.

28. Vijarania, Meenu, Vivek Jaglan, and Sonam Rani. "Conceptual Designing of Energy Efficient Optimal Path Routing Technique for Ad-hoc Network using Genetic Approach." International Journal of Computer Applications, vo.12, no.8.17, 2015.

29. Sakthivel, M., T. Gnanaprakasam, and K. Siva Krishna Rao. "Reliable data delivery in MANETs using PGF and VH scheme." In 2017 IEEE International Conference on Electrical, Instrumentation and Communication Engineering (ICEICE), pp. 1-6. IEEE, 2017.

30. Udaykumar, J., M. Sakthivel, and K. Sivakrishna Rao. "Extracting Results from Servers by using Density between Micro Clusters." International Journal of Pure and Applied Mathematics, vol. 117, no. 21, pp: 277-285, 2017.

31. Sakthivel, M., and V. Palanisamy. "Performance Enhancement By An Energy Adept Pe-Aodv With Link Failure Prediction." Journal of Theoretical \& Applied Information Technology, vol. 62, no.1, 2014.

\section{AUTHORS PROFILE}

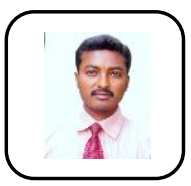

M.Sakthivel was born in India in 1982. He received the B.E. degree in Computer Science and Engineering with First Class from Adhiyamaan College of Engineering, Hosur, India, in 2003, the M.E. degree in Computer Science and Engineering with distinction from Adhiyamaan College of Engineering, Hosur, India in 2007 and the Ph.D. degree from Anna University, Chennai, India in 2016. He is currently working as Associate Professor in Computer Science and Engineering at Sree Vidyanikethan Engineering College, Tirupathi, India. His research interest include MANETs, Wireless Networks, Ad-Hoc Networks, Software Defined Networks and Cognitive Networks. He has published more than 30 papers in international journals, Conference. He is lifetime member of ISTE and IE(I).

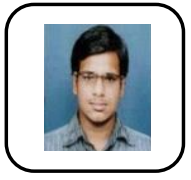

J. Uday Kumar received the B.Tech Degree with first class in Information technology from Annamcharya Institute of Technology And Sciences and received M.Tech degrees with first class in Computer Science Engineering from Sree Vidyanikethan Engineering College respectively. Currently working as Assistant Professor in Computer Science Engineering at Sree Vidyanikethan Engineering College.

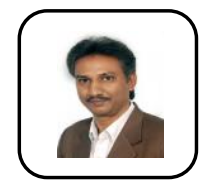

V. Saravana Kumar is working as Associate Prof in SreeNidhi Institute of Science and Technology, Hyderabad, Telangana. He received his $\mathrm{PhD}$ and M.Tech [Computer and Information Technology] from Manonmaniam Sundaranar University, Tirunelveli. He worked as Associate professor in Sree Vidyanikethan Engineering College, Tirupati, AP up to 2018. Moreover, He gained work experiences as a faculty in Centre for Bioinformatics, Pondicherry University and various Engineering Colleges in Tamilnadu. He has been publishing and presenting rich number of Research and Technical papers in International Journals, International Conferences and National Conferences. His area of interest is Digital Image Processing, Algorithm and Data mining.. 